\title{
Ensino da cirurgia ginecológica nos programas de residência médica do Brasil
}

\author{
Teaching of gynecologic surgery in residency programs of Brazil
}

\section{Editorial}

A formação do residente de Obstetrícia e Ginecologia envolve múltiplos aspectos, como o conhecimento clínico, o desenvolvimento de habilidades e técnicas, além do amadurecimento de atitudes pessoais, éticas e profissionais. Os futuros tocoginecologistas dependerão, para uma boa formação profissional, de seu esforço individual e de boas condições de treinamento oferecidas por parte das instituições formadoras, destacando-se a importância essencial da atuação dos preceptores dos programas. O treinamento durante a residência médica deve propiciar ao médico residente domínio suficiente para ingressar no competitivo mercado de trabalho de modo a permitir o atendimento necessário às suas futuras pacientes, com boa qualidade técnica e humanística.

No Brasil, são escassos os estudos abordando o ensino da Obstetrícia e da Ginecologia nos programas de residência médica. Neste fascículo, o artigo de Sória et al. ${ }^{1}$ avalia a prática da histerectomia em Serviços de Residência Médica de Obstetrícia e Ginecologia do país. Foi objetivo dos autores verificar e comparar informações sobre indicações de histerectomia para doenças uterinas benignas, vias cirúrgicas de acesso, antibioticoprofilaxia e complicações do procedimento, segundo as regiões do país. Os resultados foram similares entre os diversos Serviços avaliados que responderam ao questionário enviado. Alguns Serviços não puderam responder por falta de estrutura administrativa de registro de informações ou falta de acesso adequado aos dados solicitados, revelando dificuldades de comunicação e armazenamento de dados. Os autores destacam a falta de protocolos nacionais para a realização de histerectomias e a pouca utilização da via laparoscópica, mas concluem que a realização desta cirurgia no Brasil está em consonância com o que aponta a literatura mundial, tanto no aspecto de indicações quanto da sua execução.

Vários aspectos do ensino de nossa especialidade em programas de residência médica devem ser analisados. Um deles é o ensino da cirurgia ginecológica. O treinamento cirúrgico é um aspecto fundamental em nossa especialidade. Entretanto, não há discussão mais aprofundada do que deve ser ensinado, como e até onde deve ir a competência de um médico residente na execução dos procedimentos cirúrgicos. Este é um questionamento não só no Brasil, mas em muitos outros países, onde estudos sobre a competência adquirida de médicos residentes são feitos em maior quantidade e este assunto é uma preocupação constante da sociedade.

Professor Assistente do Departamento de Tocoginecologia da Faculdade de Ciências Médicas da Universidade Estadual de Campinas - UNICAMP - Campinas (SP), Brasil. 
O conteúdo programático de nossa especialidade ${ }^{2}$ indica a necessidade de o residente adquirir habilidades cirúrgicas para o tratamento das doenças ginecológicas benignas e malignas, incluindo urgências e emergências; adquirir habilidades em procedimentos especializados em Ginecologia, como, por exemplo, laparoscopia e histeroscopia, tanto diagnósticas quanto cirúrgicas; diagnosticar e tratar as complicações cirúrgicas mais freqüentes em Ginecologia. Faz parte dos requisitos mínimos do programa a atuação em unidades ambulatoriais, serviços de urgência e emergência, unidades de internação, centro cirúrgico e unidades de acompanhamento de pacientes graves, de forma a dar visão ampla e treinamento sobre os diferentes aspectos da cirurgia ginecológica, além do contato com procedimentos em Mastologia, Uroginecologia e Oncologia Ginecológica.

Todos sabemos como o desenvolvimento da cirurgia ginecológica mudou a face da especialidade, possibilitando o cuidado e a intervenção em muitos problemas que afetam a saúde da mulher. Os tratados de Ginecologia operatória existem há mais de um século, buscando acompanhar os avanços da ciência e da abordagem cirúrgica. Em todo este tempo, muita coisa mudou. Fístulas se tornaram mais raras. Procedimentos não realizados há dez ou 20 anos agora fazem parte do repertório da cirurgia ginecológica. Estes avanços, incluindo procedimentos endoscópicos, requerem um grau de habilidade não facilmente obtido nas possibilidades atuais de treinamento. $\mathrm{O}$ acesso à histerectomia mudou profundamente. Hoje, centenas de milhares de histerectomias são feitas em vários países, inclusive no Brasil. Richard Te Linde ${ }^{3}$ faz um comentário sobre esse fato em seu Tratado de Ginecologia Operatória: "A facilidade com que a histerectomia média pode ser realizada provou ser uma benção e uma praga para a espécie humana. Não há dúvidas de que uma histerectomia realizada com indicações apropriadas pode restabelecer a saúde de uma mulher, e até mesmo salvar sua vida. Entretanto, na prática da Ginecologia, há ampla oportunidade de observar incontáveis mulheres que foram aconselhadas a se submeter a histerectomias sem indicações apropriadas... Estou inclinado a acreditar que o maior fator isolado na promoção de histerectomias desnecessárias é uma ausência de compreensão da patologia ginecológica. A maior necessidade hoje entre aqueles que estão realizando cirurgia pélvica é um melhor conhecimento da patologia ginecológica". Neste comentário há colocações sobre lições a aprender e debates a travar. Ao cirurgião é importante conhecer a fisiopatologia das doenças ginecológicas para a boa indicação dos procedimentos cirúrgicos, fator primordial no ensino aos médicos residentes. Saber indicar uma cirurgia depende de um conhecimento que envolve estudo teórico de qualidade, ouvir profissionais mais experientes, atender pacientes, examinar ouvindo suas queixas, além da observação de resultados em mulheres operadas e não operadas, com problemas semelhantes. O conhecimento adquirido em relação à indicação cirúrgica permitirá a seleção adequada da paciente para a realização do procedimento, bem como a seleção correta da técnica operatória para cada situação. Surge, então, a necessidade do desenvolvimento das habilidades cirúrgicas durante os anos de treinamento na residência. $\mathrm{O}$ treinamento prático na execução da técnica, bem assessorado e supervisionado, permitirá a realização correta da cirurgia e orientará o necessário acompanhamento pós-operatório, sem o qual, muitas vezes, o bom trabalho pré e intra-operatório acaba sendo prejudicado, bem como a saúde da paciente, algumas vezes de forma significativa e definitiva. Mas até onde chegar nessa formação?

O campo da cirurgia pélvica sofre dos mesmos problemas relacionados ao treinamento de todos os médicos. Não é possível ensinar tudo. Nossos residentes saem preparados para realização de um número limitado de procedimentos, com raras exceções. Nossas pacientes, entretanto, continuarão tendo anomalias de desenvolvimento, prolapsos, fístulas, hemorragias, tumores, que precisarão ser tratados. O que é necessário, portanto, em termos de conhecimentos cirúrgicos, para que um médico residente, ao concluir seu programa, possa atuar satisfatoriamente, sem prejuízo a si próprio ou às suas pacientes? Em outros países, muitos educadores em Obstetrícia e Ginecologia estão preocupados com isso e destacam que o treinamento cirúrgico de residentes não é suficiente para preencher as necessidades dos futuros especialistas ${ }^{4}$. Para o Colégio Americano de Obstetrícia e Ginecologia, um dos principais objetivos para o início deste milênio é, justamente, o debate sobre a necessidade da ampliação da formação do residente de nossa especialidade, para que ele possa adquirir condições para atuar desde o nível primário até a realização de procedimentos cirúrgicos mais complexoss. Lá, os Programas de Residência Médica em Obstetrícia e Ginecologia têm duração de quatro anos, com 80 horas semanais de atividade.

Enquanto cresce o número de técnicas e métodos de abordagem cirúrgica de certos agravos, também aumenta o conhecimento no sentido de medidas mais conservadoras em várias doenças que já foram anteriormente tratadas cirurgicamente ${ }^{4}$. Citemos, por exemplo, os casos de leiomiomas uterinos, da gravidez ectópica, que dependendo de cada caso, poderão ser tratados utilizando desde métodos farmacológicos até procedimentos cirúrgicos abertos, passando por indicações laparoscópicas, dentre outras. E isto precisaria ser ensinado. 
Nas últimas duas décadas, o conceito e a prática da cirurgia minimamente invasiva têm avançado rapidamente. Esta abordagem traria, em tese, vantagens ao paciente e aos serviços de saúde. A complexidade dos procedimentos realizados laparoscopicamente tem também aumentado dramaticamente. Muitos prevêem que a laparotomia pode ser substituída no futuro pelos procedimentos chamados minimamente invasivos ${ }^{6}$. Nem todos concordam com estas afirmações e há autores que clamam por maiores evidências antes que os médicos aceitem certas afirmações e passem a adotar novos padrões de conduta, os quais podem aumentar custos, sem necessariamente melhorar resultados $^{7,8}$. Revisões sistemáticas já começam a aparecer mostrando, apesar de muita controvérsia, vantagens reais e aquelas somente aparentes quando da aplicação de novas técnicas cirúrgicas ${ }^{8}$. A formação do residente deve ser afastada de certas influências comerciais e ficar centrada no conhecimento científico de qualidade ${ }^{9,10}$. Os cirurgiões ginecológicos estão oferecendo às pacientes hoje mais que no passado, mas é necessário assegurar que mais é melhor. Se a evidência científica indicar que o caminho são os procedimentos minimamente invasivos, novamente surgirá o debate sobre até onde irá a competência necessária ao médico residente, em termos de complexidade e treinamento necessários ${ }^{11}$. O mesmo vale, por exemplo, para o treinamento em uroginecologia ${ }^{12}$, em histeroscopia, nos procedimentos endovasculares, na cirurgia oncológica minimamente invasiva. Até a robótica começa a surgir, sendo uma promessa em várias áreas cirúrgicas. Toda esta nova tecnologia, quando acessível, demandará um processo diferenciado de treinamento, não só aos residentes, mas também a seus preceptores.

Para o acompanhamento tecnológico nas áreas cirúrgicas, os programas de residência têm investido recursos consideráveis para equipar as instituições ${ }^{13}$. O custo de formação de um cirurgião é alto com esses novos requerimentos. Nos Estados Unidos, trabalho de Bridges e Diamond ${ }^{14}$ mostrou que a formação de cada residente de cirurgia custou em torno de $\$ 48.000$. Portanto, ter um programa de residência médica de qualidade em área cirúrgica implica custos, que os responsáveis pelos programas devem assumir. Por isso, é fundamental investir naquilo que realmente já está comprovado cientificamente e que é aplicável à nossa realidade.

Além destas questões, temos também dificuldades operacionais nos programas. Muitas vezes há dificuldade do residente em fazer o acompanhamento da paciente em todas as etapas: ela pode ser avaliada por um, operada por outro e revista por um terceiro. Esta perda de continuidade pode refletir em perda de qualidade de formação e do desenvolvimento do senso de integração do cuidado, algo que será cobrado de forma concreta na vida profissional. A própria atitude dos residentes para com a profissão mudou. Estudo feito por Gilbert et al. ${ }^{15}$ com residentes de Obstetrícia e Ginecologia mostrou que eles estão buscando estabelecer limites para a vida profissional, tendendo a migrar para subespecialidades que permitam rotinas com horários mais regulares e o contato com a paciente mediado pela tecnologia, sendo um grande exemplo o diagnóstico por imagem e a videoendoscopia. A paciente também mudou. Diferente de 20 anos atrás, conhece mais, escolhe mais, decide mais, levando em consideração aspectos relevantes para ela própria e não necessariamente aqueles considerados adequados pelo cirurgião. Isto ao mesmo tempo amplia a necessidade de conhecimento para a boa indicação cirúrgica, mas também da melhor técnica a ser empregada. A multiplicidade de opções pode reduzir a prática cirúrgica, com a diminuição do número de casos operados com determinada técnica, o que obriga os programas de residência médica a garantirem um número de procedimentos diversificados e suficientes para o ensino. Muitas vezes, esta dificuldade em "aprender tudo" por parte do residente e de "ensinar tudo" por parte das instituições faz com que o residente ou o egresso dos programas busquem como alternativa o treinamento cirúrgico em serviços não diretamente ligados ao ensino da residência médica, ou não regularmente cadastrados como participantes da rede de ensino de residência médica. Este é um tipo de alternativa inevitável no cenário do ensino do especialista hoje, sendo necessário conviver com esta realidade. Entretanto, da mesma forma que há necessidade de uma avaliação de qualidade dos programas oficiais de forma mais efetiva, esta também deveria ser feita nesses outros serviços.

Portanto, hoje temos um novo cenário de atuação em cirurgia ginecológica. O conhecimento, como não poderia deixar de ser, é muito diferente de dez ou 20 anos atrás. O ensino nos programas deve acompanhar esta evolução. Entretanto, será que a forma de ensinar cirurgia ginecológica mudou? Como o manuseio clínico deve ser guiado por decisões baseadas em evidências, o treinamento cirúrgico ginecológico também deve ser guiado por informação baseada em evidência, associada ao bom senso e à experiência. A aquisição e o refinamento de habilidades técnicas básicas pelo residente são as questões centrais e devem ser a primeira etapa no processo de ensino de competência cirúrgica ${ }^{16}$. O sistema de aprendizado geralmente utilizado é aprender no próprio paciente ${ }^{17}$. Muito do treinamento é feito em procedimentos cirúrgicos nos órgãos genitais, deixando de ensinar procedimentos fundamentais de cirurgia geral, necessários, principalmente, quando da ocorrência de complicações. Os programas 
de residência médica em Obstetrícia e Ginecologia devem atentar para a necessidade de ensinar técnica cirúrgica, o que implica não só conhecer os tempos operatórios de cada procedimento, mas também conhecer o instrumental cirúrgico, os fios de sutura (tipos, vantagens e desvantagens), os tipos de sutura, os diversos tipos de nós, de agulhas, os conhecimentos sobre eletrocirurgia, uso de laser e dispositivos cirúrgicos ultra-sônicos, como agredir menos os tecidos, como atuar para conter hemorragias previsíveis ou imprevisíveis, como fazer uma sutura vesical ou intestinal mais simples, desde que conhecidas as indicações e contra-indicações para tal. Para tanto, torna-se essencial algum período de contato com a Cirurgia Geral ${ }^{18}$, já obrigatório nos programas.

O treinamento do ginecologista em cirurgia requer ensino de habilidades básicas para atingir competência. Embora ninguém discorde que a melhor forma de aprender cirurgia é realizando cirurgias em pacientes e situações reais, o treinamento de muitas habilidades básicas do cirurgião ginecológico poderia ser feito utilizando outras estratégias $^{17}$, tais como vídeos, manequins, programas computadorizados, etc. Laboratórios de habilidades, já existentes para algumas especialidades, deveriam ser implantados, possibilitando ao médico residente, ao mesmo tempo que observa os procedimentos, a aquisição e o aperfeiçoamento de habilidades básicas, como dar nós e realizar suturas, o que poderia melhorar seu desempenho no campo operatório ${ }^{19,20}$. Isto seria mais um gerador de custos aos programas inicialmente, mas que devem ser minimizados com a melhor qualidade dos procedimentos realizados, melhor recuperação e menores complicações às pacientes.

Entretanto, não basta ensinar - é preciso avaliar. A educação cirúrgica, como um todo, deveria ser medida objetivamente. Habilidades básicas devem ser avaliadas e checadas após cada procedimento, o que pode auxiliar muito a prevenir omissões e erros e exercer o papel de corrigir defeitos imediatamente ${ }^{16}$. Outra grande dificuldade é saber quando o residente estará apto para executar determinado procedimento cirúrgico com segurança. Alguns tentam apontar que um número mínimo de procedimentos poderia indicar esta aptidão. Entretanto, avaliar formação falando de números de procedimentos realizados pode não ser adequado, pois a qualidade não pode ser avaliada ou julgada ${ }^{16}$. Estudo realizado nos Estados Unidos, no ano de 1996, mostrou que durante os quatro anos de duração do programa, a mediana de alguns procedimentos cirúrgicos realizados pelos médicos residentes foi a seguinte $^{21}$ : histerectomia abdominal (80 procedimentos), histerectomia vaginal (30), cirurgia para incontinência urinária (20), cirurgia para neoplasia invasora (29), cirurgia anexial (51), cirurgia para gravidez ectópica (20), laparoscopia (diagnóstica e cirúrgica) (79), cirurgia para infertilidade/endocrinopatia (32). Alguns instrumentos que avaliam a competência do cirurgião, provavelmente mais completos que a verificação isolada do número de procedimentos realizados, estão disponíveis e poderiam ser testados no Brasil, como é o caso do Objective Structured Assessment of Technical Skill (OSATS) ${ }^{22}$.

Outras qualidades mais difíceis de avaliar, mas fundamentais, como ética, profissionalismo, comunicação e liderança também devem ser buscadas e avaliadas durante o treinamento. Não basta adquirir conhecimentos e habilidades. É preciso desenvolver atitudes. Os cirurgiões ginecológicos devem compreender os aspectos psicossociais da doença ginecológica, reconhecendo a importância que cada mulher dá às suas funções orgânicas, sexuais e reprodutivas. Devem dedicar seu tempo para avaliar as pacientes apropriadamente, selecionar para cirurgia apenas as que necessitem, escolher as operações que atendem mais adequadamente às necessidades de cada uma, aprender a preservar funções e oferecer alternativas ao tratamento cirúrgico, se for possível. Destaca-se ainda a grande importância de um bom diálogo com a paciente e sua família ${ }^{23}$. Isto deve estar presente na ação dos preceptores e no cenário de ensino.

O tema da educação cirúrgica em Ginecologia transcende a residência médica. No Brasil, os tocoginecologistas não são obrigados a se submeter a exames periódicos para certificar sua competência e atualização profissional, o que torna a educação médica continuada sujeita à consciência de cada um ${ }^{24}$, embora tenhamos as iniciativas do Conselho Federal de Medicina em relação à revalidação de títulos de especialistas. Este é mais um motivo para que o ensino durante a residência médica seja eficaz e suficiente para incutir nos profissionais a necessidade de permanente aperfeiçoamento. Trata-se de questão da maior relevância porque nossa especialidade, pelo risco inerente, é a mais demandada judicialmente. Pelo resultado encontrado por Boyaciyan e Camano ${ }^{25}$, ao avaliarem o perfil dos médicos tocoginecologistas denunciados no Estado de São Paulo, não realizar residência médica implica um maior risco profissional. Falta, entretanto, avaliar a questão da qualidade da formação, pois do total de médicos denunciados, quase $40 \%$ fizeram residência. É essencial que não só os residentes sejam avaliados, mas também os programas. Outras especialidades já fazem esta avaliação rotineiramente há muitos anos, o que, ao contrário do que alguns possam imaginar, acaba fortalecendo programas e instituições e garantindo melhor formação para o futuro especialista. É necessário um comitê revisor de programas de residência médica em Obstetrícia e Ginecologia que avalie e certifique todos os 
Programas e que colabore com a avaliação feita pela Comissão Nacional de Residência Médica, principalmente em termos de controle da qualidade, destacando aspectos importantes para a especialidade.

Estas são apenas algumas das questões que consideramos importantes ao avaliar o ensino da cirurgia ginecológica nos programas de residência médica do país. Hammond e Karthigasu ${ }^{17}$ revisaram a questão do treinamento, avaliação e competências para o exercício da cirurgia ginecológica, baseados em algumas perguntas, sobre as quais sugerimos que os envolvidos com os programas de residência médica em Obstetrícia e Ginecologia do país reflitam:

1 Como tem sido o treinamento dos ginecologistas em cirurgia?

2 É necessário modificar nossos métodos de treinamento cirúrgico?

3 Que habilidades cirúrgicas são essenciais para um cirurgião ginecológico?

4 Que métodos de treinamento e avaliação de habilidades podem ser incorporados realisticamente na prática do ensino de cirurgia ginecológica?

5 Como se pode medir a competência dos residentes em realizar cirurgias ginecológicas dos mais variados tipos?

São questões importantes que merecem discussão e futuros estudos.

\section{Referências}

1. Sória HLZ, Fagundes DJ, Sória-Vieira S, Cavalli N, Santos CRC. Histerectomia e as doenças ginecológicas benignas: o que está sendo praticado na residência médica no Brasil? Rev Bras Ginecol Obstet. 2007;29(2):69-75

2. Federação Brasileira das Associações de Ginecologia e Obstetrícia (FEBRASGO) [sítio na da Internet]. Conteúdo programático para programas de residência médica em obstetrícia e ginecologia. 2006 [citado 2006 Dez 28 ]. Disponível em: http://www.febrasgo.org.br/residencia.htm.

3. Thompson JD, Warshaw J. Histerectomia. In: Rock JA, Thompson JD, editores. Te Linde ginecologia operatória. $8^{a}$ ed. Rio de Janeiro: Guanabara Koogan; 1999. p. 607-8.

4. Rogers RM Jr, Julian TM. Training the gynecologic surgeon. Obstet Gynecol. 2005; 105(1):197-200.

5. Miller FC. Ten goals for the American College of Obstetricians and Gynecologists for the first decade of the next millennium. Obstet Gynecol. 2000; 95(1): $1-5$.

6. Robertson EA, de Blok S. Decrease in the number of abdominal hysterectomies after introduction of laparoscopic hysterectomy. J Am Assoc Gynecol Laparosc. 2000; 7(4):523-5.

7. Hopkins MP. The myths of laparoscopic surgery. Am J Obstet Gynecol. 2000; 183(1):1-5.

8. Johnson N, Barlow D, Lethaby A, Tavender E, Curr E, Garry R. Surgical approach to hysterectomy for benign gynaecological disease. Cochrane Database Syst Rev. 2006; (2):CD003677

9. Jones HW Jr. Introdução. In: Rock JA, Thompson JD, editores. Te Linde ginecologia operatória. 8ª ed. Rio de Janeiro: Guanabara Koogan; 1999. p. xvii-xviii.

10. Penalver M, Mendez LE, Lambrou N. What's new in obstetrics and gynecology? J Am Coll Surg. 2002; 195(2):203-7.

11. Brill Al, Rogers RM Jr. A comprehensive program for resident training in operative laparoscopy. J Am Assoc Gynecol Laparosc. 1998; 5(3):223-8.

12. Minassian VA, Ross S, Lovatsis D, Al-Badr A, Drutz HP. Urogynecology experience in Canada's obstetrics and gynecology residency programs. Int Urogynecol J Pelvic Floor Dysfunct. 2004; 15(1):331-5.

13. Einarsson Jl, Young A, Tsien L, Sangi-Haghpeykar H. Perceived proficiency in endoscopic techniques among senior obstetrics and gynecology residents. J Am Assoc Gynecol Laparosc. 2002; 9(2):158-64.

14. Bridges M, Diamond DL. The financial impact of teaching surgical residents in the operating room. Am J Surg. 1999; 177(1):28-32.

15. Gilbert ACB, Cardoso MHCA, Wuillaume SM. Médicos residentes e suas relações com/e no mundo da saúde e da doença: um estudo de caso institucional com residentes em obstetrícia/ginecologia. Interface Comun Saúde Educ. 2006; 10(19):103-16.

16. Fenner DE. Training of a gynecologic surgeon. Obstet Gynecol. 2005; 105(1):193-6.

17. Hammond I, Karthigasu K. Training, assessment and competency in gynaecologic surgery. Best Pract Res Clin Obstet Gynaecol. 2006; 20(1):173-87.

18. Singh S, Maxwell D. Tools of the trade. Best Pract Res Clin Obstet Gynaecol. 2006; 20(1):41-59.

19. Grantcharov TP, Kristiansen VB, Bendix J, Bardram L, Rosemberg J, Funch-Jensen P. Randomized clinical trial of virtual reality simulation for laparoscopic skills training. Br J Surg. 2004; 91 (2):146-50

20. Macedonia CR, Gherman RB, Satin AJ. Simulation laboratories for training in obstetrics and gynecology. Obstet Gynecol. 2003 ; $102(2): 388-92$. 
21. Metheny WP, Ling FW, Mitchum M. What to expect from a residency program: answers from a directory of residency programs in obstetrics and gynecology. Obstet Gynecol. 1998; 91(2):311-4.

22. Martin JA, Regehr G, Reznick RK, MacRae H, Murnaghan J, Hutchison C, et al. Objective structured assessment of technical skill (OSATS) for surgical residents. Br J Surg. 1997; 84(2):273-8.

23. Rock JA. Prefácio. In: Rock JA, Thompson JD, editors. e Linde ginecologia operatória. 8ª ed. Rio de Janeiro: Guanabara Koogan; 1999. 1 p. xix.

24. Sass N, Torloni MR, Soares BGO, Atallah AN. Continuing medical education in Brazil: what about obstetricians and gynecologists? Sao Paulo Med J. 2005; 123(1):5-10.

25. Boyaciyan K, Camano L. O perfil dos médicos denunciados que exercem ginecologia e obstetrícia no Estado de São Paulo. Rev Assoc Med Bras. 2006; 52(3): 144-7. 